\title{
Enfermedad navicular con desviación axial del hueso navicular en una yegua de 33 meses de edad
}

\section{Navicular disease with axis deviation of the navicular bone in a 33 months old mare}

\author{
Jaime Alvarez $\mathrm{P}^{1 *}$ M.Sc, José Cardona Á, ${ }^{1}$ M.Sc. \\ 1 Universidad de Córdoba. Facultad de Medicina Veterinaria y Zootecnia. Departamento \\ de Ciencias Pecuarias. Montería, Colombia. *Correspondencia: jalvarezpt@hotmail.com \\ Recibido: Julio 15 de 2009; Aceptado: Diciembre10 de 2009.
}

\section{RESUMEN}

Se describe un caso de enfermedad navicular con desviación vertical del eje longitudinal del hueso navicular en una yegua de trocha pura, de 33 meses de edad, procedente del municipio de Montería (Córdoba, Colombia), a la cual se le detectó signos de cojera intermitente en el miembro posterior derecho con apoyo en lumbres, punteo constante y en abducción, aumento de volumen en el bulbo lateral de dicho miembro, marcado dolor al paso. A la palpación se determino sensibilidad en la parte posterior (bulbos y talones) del casco derecho, prueba de flexión de nudo positiva en los miembros posteriores, más marcado en el derecho, por lo que se le realizó radiología del pie y del sistema podotroclear, detectando lisis de la corteza flexora y pérdida de la diferenciación cortico-medular con desviación axial del hueso navicular. Estos hallazgos clínicos y radiológicos obedecen principalmente a enfermedad navicular. Esta patología es también conocida en equinos como síndrome navicular, la cual es una enfermedad crónica, degenerativa y progresiva, caracterizada por afectar al hueso navicular, bursa navicular o superficies adyacentes al tendón flexor digital profundo. Se realizo tratamiento farmacológico que permitió la recuperación total del paciente.

Palabras clave: Hueso navicular, bursa navicular, desviación axial, yegua.

\section{ABSTRACT}

A case of navicular disease with vertical deviation of the longitudinal axis of the navicular bone in a mare pure gauge, 33 months old, from the municipality of Monteria (Cordoba, Colombia), which was detected signs of intermittent claudication in the right posterior support in toe, tap steady and abduction, increased volume in the bulb side of the member, marking painful step. On palpation sensitivity was determined on the back (bulbs and heels) of the helmet law, bending test positive node in the hind limbs, more marked on the right, 
so it was done radiography of the foot and podotroclear system, detecting lysis of the flexor cortex and loss of cortico-medullary differentiation with axial deviation of the navicular bone. These clinical and radiological findings are mainly due to navicular disease. This condition is also known as navicular syndrome in horses, which is a chronic, degenerative and progressive, characterized by affecting the navicular bone, navicular bursa or areas adjacent to the deep digital flexor tendon. Drug treatment was performed which allowed full recovery of the patient.

Key words: Navicular bone, navicular bursa, axial desviation, mare.

\section{INTRODUCCIÓN}

La enfermedad navicular o síndrome navicular se define como una patología claudicógena bilateral, crónica, degenerativa y progresiva, que compromete al hueso navicular, la bursa navicular y las superficies adyacentes al tendón flexor digital profundo (1), es considerada una de las causas de al menos un tercio de las cojeras crónicas en equinos (2), afecta principalmente a los miembros anteriores y a nivel de la porción caudal del pie, aunque también se han reportado casos en los miembros posteriores (3).

La etiología exacta del síndrome de navicular es desconocida, sin embargo, se han propuesto mecanismos de aparición de la enfermedad que incluyen la remodelación ósea, la isquemia crónica y la bursitis (4), propiciadas por la aplicación anormal de fuerzas biomecánicas ejercidas por la segunda falange, los ligamentos naviculares o por el tendón flexor digital profundo, principalmente en animales que inician labores de trabajo a edades muy tempranas, o que poseen defectos de aplomos que impiden la regulación adecuada de las fuerzas de choque por parte de los sistemas fibroelástico y podotroclear (5).

El hueso navicular es uno de los tres huesos sesamoideos de la extremidad distal de los equinos, tiene forma de canoa y su eje longitudinal está ubicado horizontalmente, situado palmar (plantar) a la unión de las segunda y tercera falange, integrando la articulación interfalángica distal en su porción palmar (plantar). Posee dos superficies (articular y flexora), dos bordes (proximal y distal) y dos extremos (medial y lateral), está sostenido por los ligamentos sesamoideos colaterales par y el impar distalis. La bursa navicular se encuentra ubicada entre la superficie flexora del hueso navicular y el tendón flexor digital profundo (6)

El diagnóstico de la enfermedad se fundamenta en los datos obtenidos en el examen clínico, sin embargo, la confirmación se basa en los hallazgos radiológicos (7). Las proyecciones radiológicas comúnmente utilizadas para evidenciar enfermedad navicular son la dorsopalmar (DP), la lateromedial (LM) y la palmaroproximalpalmarodistal oblicua (PPPDO) (8).

Los signos radiológicos compatibles con enfermedad navicular son lísis de la corteza flexora, pérdida de la diferenciación corticomedular y remodelación de los bordes proximal y distal del hueso navicular (1)

El tratamiento recomendado de la enfermedad navicular en general está orientado en reducir el estrés del hueso, favorecer su micro circulación y retardar el proceso degenerativo de la bursa y el hueso navicular, por lo que se indica la aplicación intraarticular de una combinación de hialuronato de sodio, acetonida de triamcinolona y sulfato de amikacina. Esta opción varía con frecuencia, debido a la preferencia clínica, a la cronicidad y gravedad de la enfermedad, sin embargo esta técnica exige un riguroso protocolo antiséptico para disminuir el riesgo de infección sinovial (9). La aplicación se hace en la cara palmar (plantar) de la articulación interfalángica distal, no obstante, la localización anatómica de la bursa navicular puede hacer que las inyecciones directas, generen complicaciones tales como infección o inflamación sinovial, daño de la bursa o del 
hueso como consecuencia más grave que otras estructuras de más fácil acceso. De igual forma se debe acompañar de reposo que puede ir de semanas a meses y corrección adecuada del desbalance de aplomos del pie (10).

La enfermedad navicular es un problema común en el ámbito equino, teniendo un fuerte impacto económico, lo que la hace una patología cada vez más importante en la medicina equina (11).

\section{Caso clínico}

En el servicio clínico ambulatorio de la clínica médico-quirúrgica de grandes animales de la Facultad de Medicina Veterinaria y Zootecnia de la Universidad de Córdoba, Colombia, fue atendida una yegua, de 33 meses de edad, raza criollo colombiana, de trocha pura, con un peso aproximado de $200 \mathrm{~kg}$. Según lo reportado por los propietarios en la anamnesis, el animal tenía aproximadamente 8 meses de evidenciar cojera intermitente de los miembros posteriores, más marcada en el derecho, con aumento de volumen a nivel del bulbo lateral del mismo miembro y sin respuesta al tratamiento con fenilbutazona a dosis de $4,4 \mathrm{mg} / \mathrm{kg}$. Se le realizó examen clínico general y locomotor especializado, consistente en evaluación de los aplomos, analizando al animal en estática y en dinámica.

Al examen clínico sistemático se evidenció la presencia de cojera intermitente en el miembro posterior derecho con apoyo en lumbres, con punteo constante y aumento de volumen en el bulbo lateral de dicho miembro (Figura 1). De igual forma se encontró defecto de aplomo en los 4 cascos, bulbos y talones cerrados en los miembros posteriores, en dinámica mostró marcado dolor al paso, haciéndolo más largo, apoyándolo en lumbres y en abducción. A la palpación se determinó sensibilidad en la parte posterior (bulbos y talones) del casco derecho, prueba de flexión positiva en los miembros posteriores, más marcado en el derecho. Los demás parámetros fisiológicos se encontraban dentro de los valores

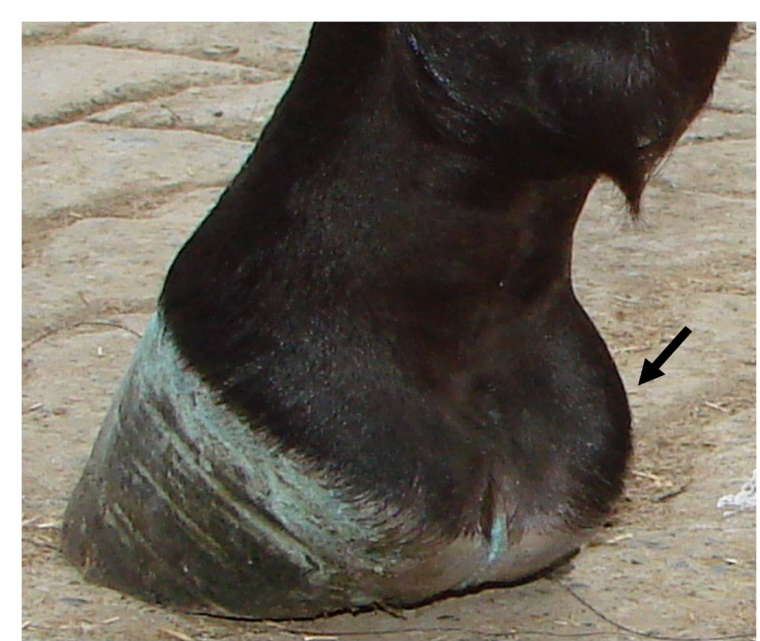

Figura 1. Aumento de volumen del bulbo lateral derecho, así como los talones cerrados y remetidos.

normales (temperatura, tiempo de llenado capilar, frecuencia cardiaca y respiratoria). También se determinó que la yegua se cruzaba de los miembros posteriores y se evidenció una herradura que tenía una turca (levantamiento externo y ventral de la herradura) a nivel de talones, con el objeto de mejorar su defecto en el andar.

Los hallazgos clínicos encontrados sugirieron un cuadro de enfermedad navicular, por lo que se recomendó un estudio radiográfico del pie y del sistema podotroclear con el fin de determinar la presencia de signos radiológicos compatibles con enfermedad navicular, el cual fue tomado en el área de clínicas de la Facultad de Medicina Veterinaria y Zootecnia de la Universidad de Córdoba, realizándose un grupo de proyecciones que incluye la LM, DP y PPPDO del pié derecho; Se detectó radiográficamente lisis de la corteza flexora (Figura 2), así como leve pérdida de la diferenciación cortico-medular y desviación axial de hueso navicular (Figura 3), confirmando la presencia de la enfermedad navicular en el animal.

Se recomendó al propietario la realización de un tratamiento médico y conservatorio, consistente en reposo por 3 meses en cama de arena, herrajes correctivos con herraduras de aluminio, con balancín y 


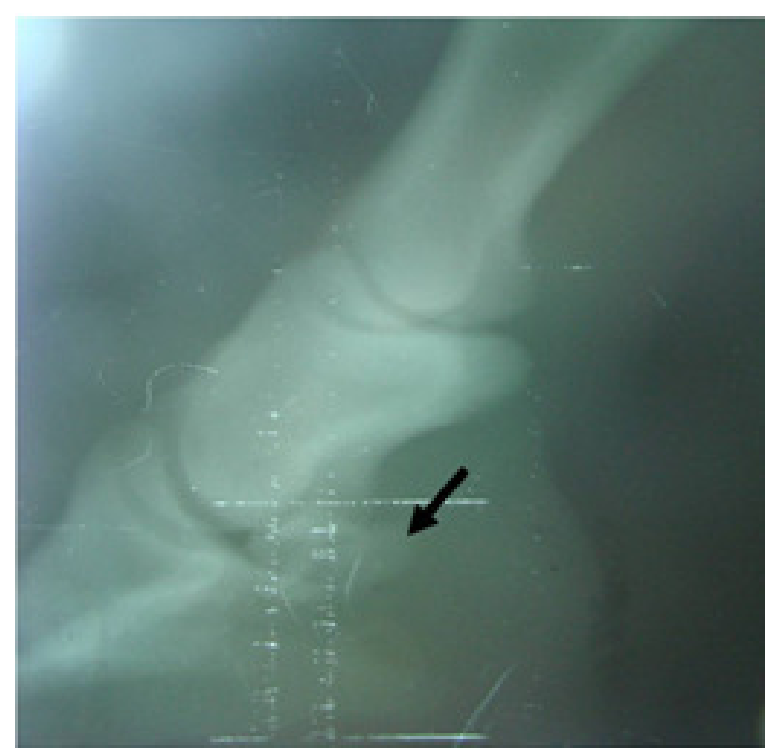

Figura 2. Radio-opacidad presente en la superficie flexora del hueso navicular, lo que indica inflamación de la bursa navicular y áreas adyacentes al TFDP, proyección LMO.

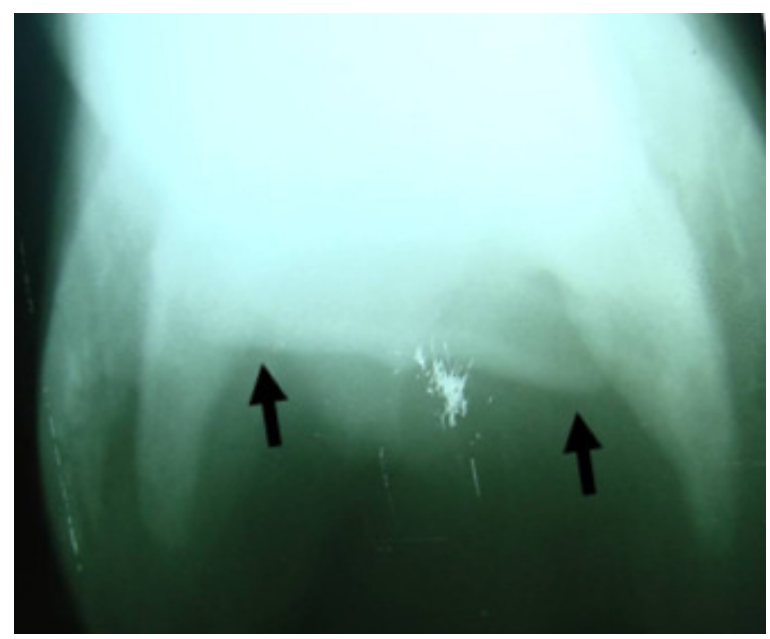

Figura 3. Pérdida de la diferenciación cortico-medular, así como la desviación vertical del eje longitudinal del hueso navicular, proyección PPPDO.

goma a los 4 cascos (Figura 4), así como la infiltración de la articulación interfalángica distal en su proyección dorsal con una aguja calibre 16 y en condiciones de asepsia, con una mezcla de $10 \mathrm{mg}$ de ácido hialurónico, $5 \mathrm{mg}$ de acetonida de triamcinolona y $40 \mathrm{mg}$ de gentamicina, en una sola aplicación.

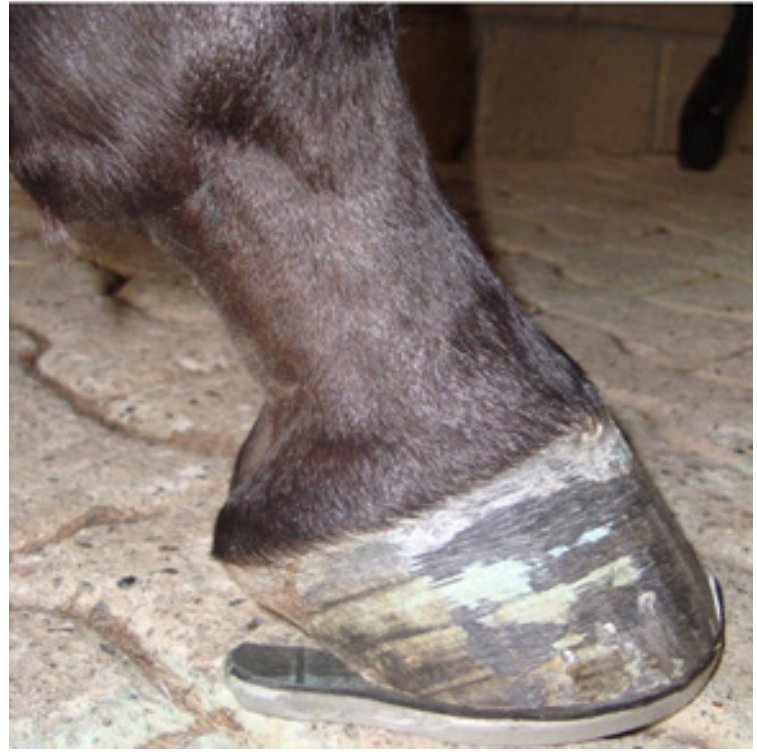

Figura 4. Herraje correctivo con herraduras de aluminio, con proyección, balancín y goma.

\section{DISCUSIÓN}

Basados en los datos anamnésicos e historia clínica, la exploración física, el examen especializado del aparato locomotor y los hallazgos radiológicos, el diagnóstico final de este paciente fue enfermedad navicular con desviación axial del hueso.

En el examen radiográfico se observó lisis de la corteza flexora en proyección LM, así como pérdida de la diferenciación cortico-medular y desviación vertical del eje longitudinal del hueso navicular en proyección PPPDO, confirmándose enfermedad navicular con desviación axial del hueso navicular. Los hallazgos radiológicos, son compatibles con los reportados por Dyson (4) y Kold y Butler (12).

Este caso se presentó en una yegua de 33 meses de edad, de trocha pura y con fines de competencia para ferias equinas, la cual había iniciado su entrenamiento a los 24 meses de edad, de igual forma tenía talones remetidos y cerrados, así como una herradura con levantamiento externo para evitar el cruzamiento de los miembros posteriores. Está reportado que este síndrome se presenta con más predisposición en equinos que inician su trabajo a edades muy tempranas y con defectos de aplomo en los cascos, ya que hay 
mayor tendencia a concusión y estrés del hueso. Tambien existe mayor presión de la segunda falange, así como mayor tensión del tendón flexor digital profundo sobre la bursa y el hueso navicular, aumentando la presión intraósea, disminuyendo su microcirculación, resultando en esclerosis y degeneración del hueso subcondral así como del cartílago articular $(5,13)$.

El tratamiento aplicado en la articulación interfalángica distal en su proyección dorsal, es un protocolo que tuvo buenos resultados clínicos. Pleasant y Crisman (5), informan que los animales tratados por esta vía mostraron marcada mejoría, posiblemente el producto aplicado llega a la bursa navicular por difusión. Sin embargo, es preciso aclarar que la bursa navicular y la articulación interfalángica distal no se comunican directamente en caballos (14), por lo que la respuesta analgésica no se muestra inmediatamente, sino después de un tiempo (10). Otro protocolo propuesto es la aplicación directa del producto en la bursa navicular. No obstante, el procedimiento requiere la penetración del tendón flexor digital profundo, por lo que si se hace en varias ocasiones puede contribuir al daño en el tendón flexor profundo y en la bursa navicular, de igual forma puede lesionar la corteza flexora del hueso navicular e incluso, podría dar lugar a importantes tendinitis y ruptura del tendón flexor profundo $(10,14)$

El hueso navicular está ubicado y sujetado en su sitio gracias a la acción de tres ligamentos naviculares, el ligamento navicular colateral par, que lo une a la segunda falange y el ligamento navicular impar distalis, que lo une a la tercera falange y le confiere estabilidad al hueso manteniendo su eje vertical en posición horizontal (14), por lo que se podría pensar que la desviación vertical, se debió al defecto de aplomo y al levantamiento externo que tenía la herradura en los talones, dificultando la regulación de las fuerzas de choque durante el apoyo por parte del sistema podotroclear, ocasionando mayor tensión del tendón flexor digital profundo y posterior fatiga de los ligamentos naviculares permitiendo su desviación, ya que está descrito que caballos con talones muy bajos, o con tacones o ramplones asimétricos, no permiten apoyar adecuadamente los talones y lograr mantener paralelo el eje longitudinal del hueso navicular (5), sin embargo no se encontraron reportes sobre esta alteración en la literatura consultada.

Se utilizó la técnica de herraje correctivo con herraduras de aluminio y goma propuesto por Gilchrist (15), debido a que reduce las fuerzas biomecánicas y el estrés del hueso, favoreciendo su recuperación.

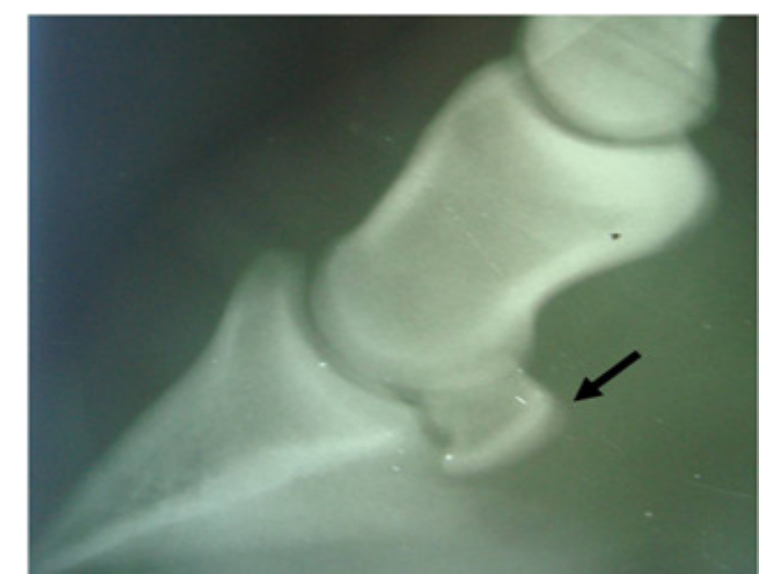

Figura 5. Apariencia radiológica normal de la superficie flexora del hueso navicular, proyección LM.

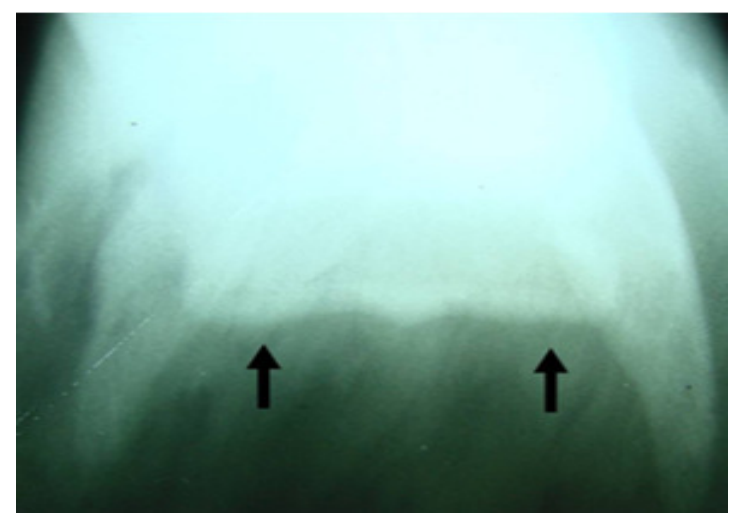

Figura 6. Apariencia radiológica normal del hueso navicular con diferenciación cortico-medular y su eje longitudinal alineado horizontalmente, proyección PPPDO.

En conclusión, el tratamiento utilizado permitio la recuperación total del paciente. Al tercer mes se encontró clínicamente sana, con ausencia de dolor en el pie y sin signos de claudicación. Al examen radiológico se determinó ausencia de radio-opacidad de la corteza flexora (Figura 5), así como la diferenciación corticomedular se notó más marcada. Sin embargo, el hallazgo radiológico más importante fue que el hueso navicular retomó su lugar y su eje longitudinal se encontró alineado horizontalmente (Figura 6). 


\section{REFERENCIAS}

1. Voute L. What can radiology tell us about palmar foot pain?. Proceedings of the 47th. Brit Eq Vet Assoc Cong $2008 ; 30-32$.

2. Dyson S, Murray R, Blunden $T$, Schramme M. Current concepts of navicular disease. Eq vet Educ 2006; 18: $45-56$.

3. Wilson A, McGuigan M, Fouracre L. McMahon $L$. The force and contact stress on the navicular bone during trot locomotion in sound horses and horses with navicular disease. Eq Vet J 2001; 33: $159-165$.

4. Dyson S. Radiological interpretation of the navicular bone. Eq Vet Educ 2008; 20: 268-280.

5. Pleasant S, Crisman M. Navicular disease in horses: pathogenesis and diagnosis. Vet Med 1997; 3: 250-257.

6. Blackwell S, Fort N. The equine foot: anatomy, trauma effects, illnesses. Proceeding of the Nor. Am Vet Conf 2007; 22-25.

7. Widmer W, Fessler J. Review: Understanding radiographic changes associated with navicular syndromeare we making progress?. Proceedings of the Am. Assoc Eq Prac 2002; 48: 155-160.

8. Martinelli M, Rantanen. The Role of Select Imaging Studies in the Lameness Examination. Proceedings of the Am Assoc Eq Prac 2002; 48: 161 - 169.
9. Carter G. Medical treatment of equine foot lameness. Proceedings of the Am Assoc Eq Prac 2009; 103 - 111.

10. Dabareiner R, Carter K, Honnas C. Injection of corticosteroids, hyaluronate, and amikacin into the navicular bursa in horses with signs of navicular area pain un responsive to other treatments: 25 cases (1999-2002) J Am Vet Assoc 2003;223: $1469-1474$.

11. Williams G. Locomotors characteristics of horses with navicular disease. Am J Vet Res 2001; 62: $206-210$.

12. Kold S, Butler J. Radiography in the horse, Foot and pastern. In Practice $2003 ; 208-215$.

13. Sandler E, Kawcak C, McIlwraith C. Remodeling of the navicular bone in response to exercise - a controlled study. Proceedings of the 4th Am Assoc Eq Pract 2001; 46 - 50.

14. Gough M, Mayhew G, Munroe G. Diffusion of mepivicaine between adjacent synovial structures in the horse. Part 1: forelimb foot and carpus. Equine Vet J 2002; 34: $80-84$.

15. Gilchrist J. Therapeutic shoeing from a farrier's perspective. Proceedings of the Am Assoc Eq Pract 2009; 168-174. 